\title{
Frobenius Method for Solving Second-Order Ordinary Differential Equations
}

\author{
Asadullah Torabi, Mohammad Alim Rohani \\ Department of Mathematics, Kandahar University, Kandahar, Afghanistan \\ Email: torabi.assad@gmail.com, alimrohani2013@gmail.com
}

How to cite this paper: Torabi, A. and Rohani, M.A. (2020) Frobenius Method for Solving Second-Order Ordinary Differential Equations. Journal of Applied Mathematics and Physics, 8, 1269-1277.

https://doi.org/10.4236/jamp.2020.87097

Received: May 28, 2020

Accepted: July 12, 2020

Published: July 15, 2020

Copyright (อ 2020 by author(s) and Scientific Research Publishing Inc. This work is licensed under the Creative Commons Attribution International License (CC BY 4.0).

http://creativecommons.org/licenses/by/4.0/

\begin{abstract}
As we know that the power series method is a very effective method for solving the Ordinary differential equations (ODEs) which have variable coefficient, so in this paper we have studied how to solve second-order ordinary differential equation with variable coefficient at a singular point $t=0$ and determined the form of second linearly independent solution. Based on the roots of initial equation there are real and complex cases. When the roots of initial equation are real then there are three kinds of second linearly independent solutions. If the roots of the initial equation are distinct complex numbers, then the solution is complex-valued.
\end{abstract}

\section{Keywords}

Regular Singular Point, Indicial Equation, Frobenius Method, Examples

\section{Introduction}

We know the linear ODEs with constant coefficients can be solved by functions known from calculus.

If a linear ordinary differential equation has variable coefficients, like Legendre's and Bessel's ODEs, it must be solved by other methods.

The power series method is a very effective method for solving the ODEs which have coefficient variable. It gives solution in the form of power series.

A power series is an infinite series of the form

$$
\sum_{r=0}^{\infty} a_{r}\left(t-t_{0}\right)^{r}=a_{0}+a_{1}\left(t-t_{0}\right)+a_{2}\left(t-t_{0}\right)^{2}+\cdots
$$

in which $t$ is a variable. $a_{0}, a_{1}, a_{2}, \cdots$ are constants, which are the coefficients of the series. $t_{0}$ is the center of the series. In particular, if $t_{0}=0$, we get a power series in power of $t$ 


$$
\sum_{r=0}^{\infty} a_{r} t^{r}=a_{0}+a_{1} t+a_{2} t^{2}+\cdots
$$

If a differential equation is given in the form

$$
t^{2} y^{\prime \prime}+t b(t) y^{\prime}+c(t) y=0
$$

Here $b(t)$ and $b(t)$ are analytic functions at $t=0$.

This equation can be written in standard form below

$$
y^{\prime \prime}+\frac{b(t)}{t} y^{\prime}+\frac{c(t)}{t^{2}} y=0
$$

If $\frac{b(t)}{t}$ and $\frac{c(t)}{t^{2}}$ are analytic at $t=0$, then the solution of the equation will be analytic at $t=0$, which can be represented in the form

$$
y(t)=\sum_{r=0}^{\infty} a_{r} t^{r}
$$

However if either $\frac{b(t)}{t}$ or $\frac{c(t)}{t^{2}}$ are not analytic at $t=0$, in other words we have a singular point at $t=0$. Then solution cannot be represented in the series, so we must go to power series expanded method which is called Frobenius method.

The Frobenius method enables us to solve such types of differential equations for example, Bessel's equation

$$
y^{\prime \prime}+\frac{1}{t} y^{\prime}+\left(\frac{t^{2}-v^{2}}{t^{2}}\right) y=0, \quad(v \text { is parameter })
$$

of the form of "Equation (4)". Here $b(t)=1$ and $c(t)=t^{2}-v^{2}$ are analytic at $t=0$. This ODE could not be solved by power series method, and it requires the Frobenius method.

P. Haarsa and S. Pothat have considered such types of ODEs, but they acquired exclusively the first solution besides general solution. Similarly, Anil Hakim Syofra, Rika Permatasari and Lily Adriani Nazara attained the form of the second solution in real case of the mentioned equations in their research paper.

1) We will study how we can solve second order ODEs at a singular point.

2) Discuss the real and complex cases of the solution with examples.

\section{Regular and Singular Point}

A regular point of

$$
y^{\prime \prime}+p(t) y^{\prime}+q(t) y=0
$$

is a point $t_{0}$ in which the coefficients $p(t)$ and $p(t)$ are analytic functions. Likewise, a regular point of the ordinary differential equation

$$
\tilde{h}(t) y^{\prime \prime}+\tilde{p}(t) y^{\prime}+\tilde{q}(t) y=0
$$

is $t_{0}$ in which $\tilde{h}, \tilde{p}$ and $\tilde{q}$ are analytic and $\tilde{h}\left(t_{0}\right) \neq 0$ (divide by $\tilde{h}$ we get the standard form). So the power series method can be applied. If $t_{0}$ is not a 
regular point, it is called a singular point [1] [2].

\section{Frobenius Method}

If $t=0$ is a singular point of the ordinary differential "Equation (4)", then it has at least one solution of the form

$$
y(t)=t^{k} \sum_{r=0}^{\infty} a_{r} t^{r}=t^{k}\left(a_{0}+a_{1} t+a_{2} t^{2}+\cdots\right), a_{0} \neq 0
$$

in which $k$ may be any (real or complex) number [3].

The second-order differential "Equation (4)" also has a second solution which may be similar to solution one with a different $k$ and different coefficients, or may have a logarithmic term. Solutions one and two are linearly independent [2] [4].

\section{Indicial Equation}

Now we shall discuss the method of Frobenius for solving "Equation (4)" at a singular point $t=0$. Multiply "Equation (4)" by $t^{2}$, we get

$$
t^{2} y^{\prime \prime}+t b(t) y^{\prime}+c(t) y=0
$$

Now $b(t)$ and $c(t)$ are expanding in power series,

$$
b(t)=b_{0}+b_{1} t+b_{2} t^{2}+\cdots \text { and } c(t)=c_{0}+c_{1} t+c_{2} t^{2} \cdots
$$

Differentiating "Equation (6)" term by term, finding

$$
\left.\begin{array}{l}
y^{\prime}(t)=\sum_{r=0}^{\infty}(r+k) a_{r} t^{r+k-1}=t^{k-1}\left[k a_{0}+(k+1) a_{1} t\right] \\
y^{\prime \prime}(t)=\sum_{r=0}^{\infty}(r+k)(r+k-1) a_{r} t^{r+k-2}=t^{k-2}\left[k(k-1) a_{0}+(k+1) k a_{1} t+\cdots\right]
\end{array}\right\}
$$

By putting the valves of $y(t), y^{\prime \prime}(t)$ and $y^{\prime \prime}(t)$ into "Equation (7)", we readily obtain

$$
\begin{aligned}
& t^{k}\left[k(k-1) a_{0}+\cdots\right]+\left(b_{0}+b_{1} t+\cdots\right) t^{k}\left(k a_{0}+\cdots\right) \\
& +\left(c_{0}+c_{1} t+\cdots\right) t^{k}\left(a_{0}+a_{1} t+\cdots\right)=0
\end{aligned}
$$

equating the sum of the coefficients of each power of $t^{k}, t^{k+1}, t^{k+2}, \cdots$ to zero.

This gives a structure of equations with the unknown coefficients $a_{r}$.

The corresponding equation to the power $t^{k}$ is

$$
\left[k(k-1)+b_{0} k+c_{0}\right] a_{0}=0
$$

Since by assumption that $a_{0} \neq 0$ the above expression must be zero. This gives

$$
k(k-1)+b_{0} k+c_{0}=0
$$

This equation is important and is known as indicial equation of the ordinary differential "Equation (4)". It plays the role as follows:

Method of Frobenius gives a basis of solution. One solution of the given ODE 
will be of the form of "Equation (6)", where $k$ is a root of "Equation (10)". The second one will be of the form specified by the indicial equation [1] [2].

Let the roots of indicial equation be $k_{1}$ and $k_{2}$. Then we have:

The Real Case: suppose $k_{1}$ and $k_{2}$ are real and $k_{1}>k_{2}$. There are three cases as follows:

Case 1: Distinct roots not different by an integer

If $k_{1}$ and $k_{2}$ are such that $\left(k_{1}-k_{2}\right)$ is not an integer. Then we have

$$
y_{1}(t)=t^{k_{1}}\left(a_{0}+a_{1} t+a_{2} t^{2}+\cdots\right)
$$

and

$$
y_{2}(t)=t^{k_{2}}\left(A_{0}+A_{1} t+A_{2} t^{2}+\cdots\right)
$$

with coefficients obtained successively from "Equation (9)" in which $k=k_{1}$ and $k=k_{2}$ respectively [1] [2] [4].

\section{Example 1:}

We solve the ODE

$$
t^{2} y^{\prime \prime}+1.5 t y^{\prime}-0.5 y=0
$$

Its standard form is

$$
y^{\prime \prime}+1.5 \frac{1}{t} y^{\prime}+0.5 \frac{1}{t^{2}} y=0
$$

Substitute $y=t^{k}$ and its derivatives $y^{\prime}=k t^{k-1}$ and $y^{\prime \prime}=k(k-1) t^{k-2}$ into the above equation, we get

$$
k(k-1) t^{k-2}+1.5 \frac{1}{t} k t^{k-1}-0.5 \frac{1}{t^{2}} y=0
$$

We have the axillary equation

$$
k(k-1)+1.5 \frac{1}{t} k-0.5=0
$$

or

$$
k^{2}+0.5 k-0.5=0
$$

0.5 and -1 are the roots. Hence tow solutions for all positive $t$ is $y_{1}=t^{\frac{1}{2}}$ and $y_{2}=\frac{1}{t}[2][5]$.

Case 2: Double roots $\left(k_{1}=k_{2}=k\right)$

A basis is

$$
y_{1}(t)=t^{k}\left(a_{0}+a_{1} t+a_{2} t^{2}+\cdots\right)
$$

In case tow we must have algorithm, where in case three we may or may not. Therefore the second solution is

$$
y_{2}(t)=y_{1}(t) \ln t+t^{k}\left(A_{0}+A_{1} t+A_{2} t^{2}+\cdots\right), t>0
$$

Example 2:

We solve the ODE

$$
t(t-1) y^{\prime \prime}+(3 t-1) y^{\prime}+y=0
$$


Writing "Equation (15)" in standard form of "Equation (4)"

$$
y^{\prime \prime}+\frac{3 t-1}{t(t-1)} y^{\prime}+\frac{1}{t(t-1)} y=0
$$

We see that it satisfies the mentioned condition, by putting "Equation (6)" and its derivatives into "Equation (15)", we get

$$
\begin{aligned}
& \sum_{r=0}^{\infty}(r+k)(r+k-1) a_{r} t^{r+k}-\sum_{r=0}^{\infty}(r+k)(r+k-1) a_{r} t^{r+k-1} \\
& +3 \sum_{m=0}^{\infty}(r+k) a_{r} t^{r+k}-\sum_{m=0}^{\infty}(r+k) a_{r} t^{r+k-1}+\sum_{r=0}^{\infty} a_{r} t^{r+k}=0
\end{aligned}
$$

The slightest power is $t^{k-1}$, occurring in the second and the fourth series; by associating the sum of its factors to zero, we have

$$
[-k(k-1)-k] a_{0}=0
$$

Thus $k^{2}=0$.

Therefore this indicial equation has the paired roots $k=0$ [2] [4].

First solution

By addition the value of $k=0$ in (16) and compare the sum of the power $t^{s}$ to zero, we attain

$$
s(s-1) a_{s}-(s+1) s a_{s+1}+3 s a_{s}-(s+1) a_{s+1}+a_{s}=0
$$

$a_{s+1}=a_{s}$. Hence $a_{0}=a_{1}=a_{2}=\cdots=0$. And by selecting $a_{0}=1$, we get the answer

$$
y_{1}(t)=\sum_{r=0}^{\infty} t^{r}=\frac{1}{1-t} \quad(|t|<1)
$$

\section{Second solution}

For second independent solution, we apply the method of decrease of order, replacing $y_{2}=u y_{1}$ and its derivatives into equation. We have $p=\frac{3 t-1}{t(t-1)}$, the
factor of $y^{\prime}$ in (15) in standard form.

By partial fractions,

$$
-\int p \mathrm{~d} t=-\int \frac{3 t-1}{t(t-1)} \mathrm{d} t=-\int\left(\frac{2}{t-1}+\frac{1}{t}\right) \mathrm{d} t=-2 \ln (t-1)-\ln t
$$

Hence

$$
\begin{gathered}
u^{\prime}=U=y_{1}^{-2} \mathrm{e}^{-\int p \mathrm{~d} t}=\frac{(t-1)^{2}}{(t-1)^{2} t}=\frac{1}{t}, u=\ln t \\
y_{2}=u y_{1}=\frac{\ln t}{1-t}
\end{gathered}
$$

These functions are linearly independent and thus form a basis on the interval $0<x<1$ (as well as on $1<x<\infty$ ) [2] [5] [6].

Case 3: $k_{1}-k_{2}$ is a positive integer

The tow solutions are

$$
y_{1}(t)=t^{k_{1}}\left(a_{0}+a_{1} t+a_{2} t^{2}+\cdots\right)
$$




$$
y_{2}(t)=k y_{1}(t) \ln t+t^{k_{2}}\left(A_{0}+A_{1} t+A_{2} t^{2}+\cdots\right)
$$

\section{Example 3:}

We solve the following ODE

$$
\left(t^{2}-t\right) y^{\prime \prime}-t y^{\prime}+y=0
$$

Replacing "Equation (6)" and "Equation (8)", in "Equation (19)", we get

$$
\left(t^{2}-t\right) \sum_{r=0}^{\infty}(r+k)(r+k-1) a_{r} t^{r+k-2}-t \sum_{r=0}^{\infty}(r+k) a_{r} t^{r+k-1}+\sum_{r=0}^{\infty} a_{r} t^{r+k}=0
$$

By taking $t^{2}, t$ and $t$ exclusive the summations and bring together all terms with power $t^{r+k}$ and simplify algebraically,

$$
\sum_{r=0}^{\infty}(r+k-1)^{2} a_{r} t^{r+k}-\sum_{r=0}^{\infty}(r+k)(r+k-1) a_{r} t^{r+k-1}=0
$$

We set $r=s$ in the first series and $r=s+1$ in the second series, thus $s=r-1$. Then

$$
\sum_{s=0}^{\infty}(s+k-1)^{2} a_{s} t^{s+k}-\sum_{s=0}^{\infty}(s+k+1)(s+k) a_{s+1} t^{s+k}=0
$$

Here the lowest power is $t^{k-1}$ and provides the indicial equation

$$
k(k-1)=0
$$

$k_{1}=1$ and $k_{2}=0$ are the roots, they are different by an integer.

\section{First solution}

From (20) with $k=k_{1}=1$, we have

$$
\sum_{s=0}^{\infty}\left[s^{2} a_{s}-(s+2)(s+1) a_{s+1}\right] t^{s+1}=0
$$

From this we get the recurrence relation

$$
a_{s+1}=\frac{s^{2}}{(s+2)(s+1)} a_{s} \quad(s=0,1, \cdots)
$$

Therefore $a_{1}=0, a_{2}=0, \cdots$ consecutively.

For $a_{0}=1$, we get first solution as $y_{1}=t^{k_{1}} a_{0}=t$.

\section{Second solution}

Applying the method of reduction of order, we replace $y_{2}=y_{1} u=t u$, $y_{2}^{\prime}=t u^{\prime}+u$ and $y_{2}^{\prime \prime}=t u^{\prime \prime}+2 u^{\prime}$ into the ODE (19), we have

$$
\left(t^{2}-t\right)\left(t u^{\prime \prime}+2 u^{\prime}\right)-t\left(t u^{\prime}+u\right)+t u=0
$$

Drops out $t u$. Division by $t$ and simplification gives

$$
\left(t^{2}-t\right) u^{\prime \prime}+(t-2) u^{\prime}=0
$$

From partial fractions and integrating, we obtain

$$
\frac{u^{\prime \prime}}{u^{\prime}}=-\frac{t-2}{t^{2}-t}=-\frac{2}{t}+\frac{1}{t-1}, \ln u^{\prime}=\ln \left|\frac{t-1}{t^{2}}\right|
$$

By taking exponents and integrating again, we get

$$
\begin{aligned}
& u^{\prime}=\frac{t-1}{t^{2}}=\frac{1}{t}-\frac{1}{t^{2}}, \quad u=\ln t+\frac{1}{t} \\
& y_{2}=t u=t \ln t+1
\end{aligned}
$$


These two solutions are linearly independent, and $y_{2}$ has logarithmic term [2] [6].

The Complex Case: If the roots of the indicial equation are distinct complex Numbers, $k$ and $\bar{k}$ say, then the solution is a complex-valued and is of the form

$$
y(t)=t^{k} \sum_{r=0}^{\infty} a_{r} t^{r}
$$

in which the coefficients $a_{r}$ may be complex. $y_{1}(t)$ and $y_{2}(t)$ are the real and imaginary parts of $y(t)$, respectively and are linearly independent [1].

Example 4:

$$
t^{2}(t+1) y^{\prime \prime}+t y^{\prime}+(t+1)^{3} y=0
$$

Replacing "Equation (6)" and "Equation (8)", in "Equation (22)”, we obtain

$$
t^{2}(t+1) \sum_{r=0}^{\infty}(r+k)(r+k-1) a_{r} t^{r+k-2}+t \sum_{r=0}^{\infty}(r+k) a_{r} t^{r+k-1}+(t+1)^{3} \sum_{r=0}^{\infty} a_{r} t^{r+k}=0
$$

or

$$
\begin{aligned}
& \sum_{r=1}^{\infty}(r+k-1)(r+k-2) a_{r-1} t^{r+k}+\sum_{r=0}^{\infty}(r+k)(r+k-1) a_{r} t^{r+k} \\
& +\sum_{r=0}^{\infty}(r+k) a_{r} t^{r+k}+\sum_{r=3}^{\infty} a_{r-3} t^{r+k}+\sum_{r=2}^{\infty} 3 a_{r-2} t^{r+k}+\sum_{r=1}^{\infty} 3 a_{r-1} t^{r+k}+\sum_{r=0}^{\infty} a_{r} t^{r+k}=0
\end{aligned}
$$

We know the initial equation is

$$
k(k-1)+k+1=0
$$

or

$$
k^{2}+1=0
$$

Its roots $k= \pm i$. Thus, there is a complex-valued solution.

Since the index of the sums has unlike starting points, we separate the cases $r=0, r=1, r=2$ and $r \geq 3$ for getting the following:

$$
\begin{gathered}
r=0 \quad\left(k^{2}+1\right) a_{0}=0, \\
r=1 \quad\left[(k+1)^{2}+1\right] a_{1}+[k(k-1)+3] a_{0}=0, \\
r=2\left[(k+2)^{2}+1\right] a_{2}+[(k+1) k+3] a_{1}+3 a_{0}=0, \\
r \geq 3 \quad\left[(k+r)^{2}+1\right] a_{r}+[(k+r-1)(k+r-2)+3] a_{r-1}+3 a_{r-2}+a_{r-3}=0 .
\end{gathered}
$$

Then $r=0$ case implies that $k= \pm i$, as standard, $a_{0}$ is arbitrary but nonzero.

Substituting $k=i$ (the $k=-i$ case will give same result) and $a_{0}=1$ into the cases $r=1$ and $r=2$ above, gives $a_{1}=i$ and $a_{2}=-\frac{1}{2}$. The general recurrence relation is

$$
\left[(i+r)^{2}+1\right] a_{r}+[(i+r-1)(i+r-2)+3] a_{r-1}+3 a_{r-2}+a_{r-3}=0
$$


From which $a_{r}=\frac{i^{r}}{r !}$.

It follows that

$$
y(t)=t^{i} \sum_{r=0}^{\infty} \frac{i^{r}}{r !} t^{r}=t^{i} \mathrm{e}^{i t}
$$

Suppose $t>0$, Therefore, we can write $t^{i}=\mathrm{e}^{i \ln t}$ and $y(t)=\mathrm{e}^{i(t+\ln t)}$. By using Euler's formula, the real and imaginary parts are

$$
\begin{aligned}
& y_{1}(t)=\cos (t+\ln t) \\
& y_{2}(t)=\sin (t+\ln t)
\end{aligned}
$$

\section{Result}

There is at least one Frobenius solution, in each case. When the roots of initial equation are real, there is a Frobenius solution for the larger of the two roots. If $y_{1}$ is a Frobenius solution and there is not a second solution, then a second independent solution is the sum of a logarithmic expression $y_{1}(t) \ln t$ and a Frobenius series. If there are complex roots, the first and second solutions will be of the real and imaginary parts of $y(t)$.

\section{Conclusions}

When $t=0$ is a singular point of the second-order ordinary differential equation

$$
y^{\prime \prime}+\frac{b(t)}{t} y^{\prime}+\frac{c(t)}{t^{2}} y=0
$$

in which $b(t)$ and $c(t)$ are not analytic in $t=0$, the Frobenius method will apply and it has at least one solution which can be represented in the form

$$
y(t)=t^{k} \sum_{r=0}^{\infty} a_{r} t^{r}=t^{k}\left(a_{0}+a_{1} t+a_{2} t^{2}+\cdots\right)
$$

The above ordinary differential equation also has a second solution such that they are linearly independent.

Its form will be specified by "Equation (6)" in the following cases.

The real case: If the roots of the initial equation are real, then there are the following cases:

Case 1: $k_{1}-k_{2}$ is not an integer

$$
y_{2}(t)=t^{k_{2}}\left(A_{0}+A_{1} t+A_{2} t^{2}+\cdots\right)
$$

Case 2: double roots $k_{1}=k_{2}=k$

$$
y_{2}(t)=y_{1}(t) \ln t+t^{k}\left(A_{1} t+A_{2} t^{2}+\cdots\right)
$$

Case 3: roots differing by an integer

$$
y_{2}(t)=k y_{1}(t) \ln t+t^{k_{2}}\left(A_{0}+A_{1} t+A_{2} t^{2}+\cdots\right)
$$

The complex case: When the roots of initial equation are distinctly complex, solution is of the form 


$$
y(t)=t^{k} \sum_{r=0}^{\infty} a_{r} t^{r}
$$

in which the coefficients $a_{r}$ may be complex. $y_{1}(t)$ and $y_{2}(t)$ are the real and imaginary parts of $y(t)$, respectively.

\section{Conflicts of Interest}

The authors declare no conflicts of interest regarding the publication of this paper.

\section{References}

[1] Adkins, W. and Davidson, M.G. (2010) Ordinary Differential Equation. Springer, New York.

[2] Kreyszig, E. (2006) Advance Engineering Mathematics. 9th Edition, Willy Ltd., India.

[3] Haarsa, P. and Pothat, S. (2014) The Frobenius Method on a Second Order Homogeneous Linear ODEs. Advance Studies in Theoretical Physics, 8, 1145-1148. https://doi.org/10.12988/astp.2014.4798

[4] Syofra, A.H., Permatasari, R. and Nazara, A. (2016) The Frobenius Method for Solving Ordinary Differential Equation with Coefficient Variable. IJSR, 5, 2233-2235. https://www.ijsr.net/search index results_paperid.php?id=ART2016719 https://doi.org/10.21275/v5i7.art2016719

[5] Hartman, P. (2002) Ordinary Deferential Equation. 2nd Edition, SIAM, Philadelphia.

[6] Birkhoff, G. and Rota, G.-C. (1989) Ordinary Differential Equation. 4th Edition, Wily, New York. 